\title{
Comparative Pharmacokinetics of Gallic Acid After Oral Administration of Gallic Acid Monohydrate in Normal and Isoproterenol-Induced Myocardial Infarcted Rats
}

\section{OPEN ACCESS}

Edited by:

Ruiwen Zhang,

University of Houston, United States

Reviewed by:

Kaisar Raza,

Central University of Rajasthan, India

Song Gao,

Texas Southern University,

United States

*Correspondence:

Hua Li

lihuasmile@aliyun.com

Bang-Le Zhang

blezhang@fmmu.edu.cn

Si-Wang Wang

wangsiw@fmmu.edu.cn

tThese authors have contributed equally to this work.

Specialty section:

This article was submitted to

Ethnopharmacology,

a section of the journal

Frontiers in Pharmacology

Received: 22 January 2018 Accepted: 21 March 2018

Published: 06 April 2018

Citation:

Yu Z, Song F, Jin $Y-C$, Zhang $W-M$,

Zhang Y, Liu E-J, Zhou D, Bi L-L,

Yang $Q, L i H$, Zhang $B-L$ and

Wang S-W (2018) Comparative

Pharmacokinetics of Gallic Acid After

Oral Administration of Gallic Acid

Monohydrate in Normal

and Isoproterenol-Induced Myocardial

Infarcted Rats.

Front. Pharmacol. 9:328.

doi: 10.3389/fphar.2018.00328
Zhe Yu't, Fan Song ${ }^{2 t}$, Yu-Chen Jin ${ }^{2,3,4 t}$, Wei-Min Zhang ${ }^{2,3 t}$, Ya Zhang ${ }^{2}$, En-Jun Liu' ${ }^{2}$, Dan Zhou ${ }^{2,5}$, Lin-Lin Bi', Qian Yang ${ }^{2}$, Hua Li ${ }^{2 *}$, Bang-Le Zhang ${ }^{4 *}$ and Si-Wang Wang ${ }^{2 *}$

${ }^{1}$ Department of Pharmaceutical Analysis, School of Pharmacy, Fourth Military Medical University, Xi'an, China, ${ }^{2}$ Department of Natural Medicine, School of Pharmacy, Fourth Military Medical University, Xi'an, China, ${ }^{3}$ Cadet Brigade, Fourth Military Medical University, Xi'an, China, ${ }^{4}$ Department of Pharmaceutics, School of Pharmacy, Fourth Military Medical University, Xi'an, China, ${ }^{5}$ Department of Pharmacy, Ninth Hospital of Xi'an, Xi'an, China

Gallic acid (GA) is a polyphenolic natural product widely distributed in food, beverage, and traditional Chinese herbs with beneficial effects on the cardiovascular system. In this research, a comparative study was conducted to investigate the possible difference of pharmacokinetic process in normal and isoproterenol-induced myocardial infarcted rats after oral administration of GA monohydrate with the dose of 50 and $100 \mathrm{mg} / \mathrm{kg}$, respectively. Quantification of GA in rat plasma was achieved by using a simple and rapid high-performance liquid chromatographic method. The results revealed that pharmacokinetics of GA were greatly different between normal and pathological state. GA exhibited slower absorption into the bloodstream, and yielded 1.7 -fold $(50 \mathrm{mg} / \mathrm{kg}$ GA) and 1.3-fold (100 mg/kg GA) less values of area under concentration-time curve as well as 2.5-fold lower of maximum blood concentration $\left(\mathrm{C}_{\max }\right)$ in $\mathrm{Ml}$ rats than those in normal rats. In addition, significant prolonged $T_{1 / 2}$ and MRT as well as decreased $\mathrm{CL}$ were also registered in $\mathrm{Ml}$ rats. Our findings suggest that myocardial infarction could alter the pharmacokinetic process of GA, and thus the potential pharmacokinetic differences of herbal preparations (or dietary nutrition) containing GA between normal and pathological conditions should be brought to the forefront seriously in clinical practice.

Keywords: gallic acid, pharmacokinetics, myocardial infarction, isoproterenol, HPLC

\section{INTRODUCTION}

Ischemic heart disease (IHD) is the most common cause of death in the world, taking 100s of millions of lives away over the decades. In recent years, the prevention of IHD has been associated with the ingestion of fresh fruits or herbs rich in natural antioxidants (Farías et al., 2017). The protective effects of plants can be due to the presence of flavonoids, anthocyanins, and phenolic compounds. Gallic acid (GA) (3,4,5-trihydroxybenzoic acid, Figure 1) is an endogenous plant polyphenol product (phenolic acid) which richly distributed in at least 12 plant species including Rheum palmatum L., Rosa chinensis Jacq., and Paeonia suffruticosa Andr. (Kosuru et al., 2017). 
Also, as the active phenolic acid compound of many traditional Chinese herbs and herbal preparations, e.g., Cortex Moutan and ShuangDan Oral Liqued (Li et al., 2011), GA is reported to have memorably cardioprotective activity and receiving considerable attention in medical and nutritional research. A previous study has revealed that GA has beneficial effect on lysosomal enzymes, lipid peroxidation, and reduced glutathione in isoproterenol induced cardiac damage in rats (Stanely et al., 2009). Another report showed that GA could protect the heart by directly combination with free radicals and lead to inactivate them, which may suppress the intracellular concentration of free radicals (Priscilla and Prince, 2009). In addition, GA was proved to present negative chronotropic and inotropic effects in isoproterenol induced myocardial damage, which is similar to propranolol (Nwokocha et al., 2017).

Consistently with the extensive research into the cardioprotective activities of GA, a few reports had addressed the disposition profiles of GA in vivo. C8-modified graphene@mSiO2 composites based method and UPLC-MS/MS method have been applied to the kinetic studies of GA (Ma et al., 2015; Xu et al., 2017; Zhu et al., 2017). However, most of these work were carried out with the administration of crude drugs and/or Chinese patented medicines which content GA. Only in a few work, e.g., the previous studies of Shahrzad and Bitsch (1998), Yasuda et al. (2000), and Shahrzad et al. (2001), metabolic and primary pharmacokinetic properties of GA were studied with high-performance liquid chromatography (HPLC) methods after oral administration of GA monomer. Further, little work has been carried out to investigate the effects of ischemic condition on pharmacokinetics of GA and their related mechanisms. As we all know, remedies are used to treat diseases and in most cases patients are the ultimate consumers of them. It is possible that drug metabolic enzymes, transporters, cell membrane permeability and the change of microbe group could be affected by physiological and pathological changes, which enable the pharmacokinetics of GA in the body to be altered, including the process of absorption, distribution, metabolism, and excretion.

Therefore, the aim of our study was to investigate the possible difference of pharmacokinetic process in normal and isoproterenol-induced myocardial infarcted rats after oral administration of GA monohydrate. Pharmacokinetic data could

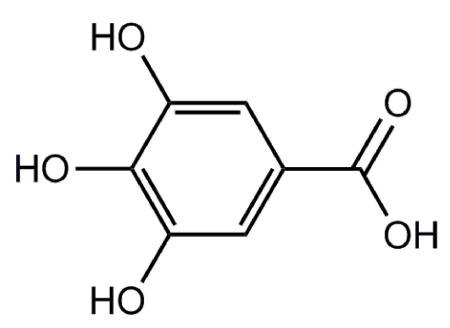

FIGURE 1 | Chemical structures of gallic acid (GA) (chemical name: 3,4,5-trihydroxybenzoic acid). provide additional information on preclinical and clinical use of herbal preparations as well as dietary nutrition comprising GA.

\section{MATERIALS AND METHODS}

\section{Materials and Reagents}

Gallic acid monohydrate (99.0\%, purity) was supplied by Jin Lv Biotech. Co., Ltd. (Xi'an, China); reference compound of GA (99.0\%, purity) was purchased from the National Institute for the Control of Pharmaceutical and Biological Products (Beijing, China); 4-acetamidophenol (99.0\%, purity) used as internal standard (IS) was purchased from the SinoPharm Chemical Reagent, Co., Ltd. (Shanghai, China). Isoproterenol hydrochloride was purchased from Tokyo Chemical Industry, Co., Ltd. (Kita-Ku, Tokyo, Japan). Methanol (HPLC grade) was obtained from Duksan Pure Chemicals (Ansan, South Korea). Glacial acetic acid (HPLC grade) was obtained from Mallinckrodt Baker (Phillipsburg, NJ, United States). The deionized water was prepared from Millipore water purification system (Milford, MA, United States) and was filtered with a $0.22-\mu \mathrm{m}$ membrane. Other biochemical reagents and chemicals were all of analytical grade.

\section{Animals}

Male Sprague-Dawley rats were purchased from Experimental Animal Research Center, the Fourth Military Medical University (Xi'an, China). They were kept in the individually ventilated cages (IVCs) system under controlled experimental conditions of $12 \mathrm{~h}$-light/dark cycle for at least 5 days before starting the experiments and fed with standard laboratory food and water ad libitum. The rats (320 350 g) were fasted (except for water) for $12 \mathrm{~h}$ prior to the experiment of oral administration of GA. Animal experiments were carried out in accordance with the policies and guidelines of Institutional Animal Care and Use Committee (IACUC) of the Fourth Military Medical University. The experimental protocols involving animals were reviewed and approved by the IACUC of the Fourth Military Medical University (Ethical Clearance No. KY2016-3034-1). The animal welfare, care, and use were managed by professionally welltrained technical personnel with relative educational background. All efforts were made to minimize animal suffering and to reduce the number of animals used.

\section{Induction of Myocardial Injury}

Myocardial injury was induced in model group by subcutaneous (s.c.) injection of $85 \mathrm{mg} \mathrm{kg}^{-1}$ of ISO daily for 2 consecutive days to rats ( $\mathrm{Li}$ et al., 2012, 2016). After $12 \mathrm{~h}$ of last dose of ISO administration, rats were anesthetized with pentobarbital sodium (35 $\mathrm{mg} \mathrm{kg}^{-1}$, i.p.), blood was collected by abdominal aorta and allowed to clot for $1 \mathrm{~h}$ at room temperature. Serum was subsequently separated by centrifugation at $4000 \mathrm{rpm}$ for $15 \mathrm{~min}$ and stored at $-80^{\circ} \mathrm{C}$ for biochemical assays. After blood collection, rats were sacrificed by cervical decapitation. Heart and small intestine tissue was excised and rinsed immediately in icecold normal saline, then frozen in liquid nitrogen and stored at $-80^{\circ} \mathrm{C}$ for further analysis. 


\section{Assay of Serum Myocardial Injury Markers}

Activity of serum creatine kinase-MB (CK-MB) was measured using commercial kit (Biosino Bio-technology and Science, Inc., Beijing, China). Serum levels of cardiac troponin I (cTnI) was estimated using standard commercial kits (Jiancheng Biotechnology and Science, Inc., Nanjing, China).

\section{Triphenyl Tetrazolium Chloride (TTC) and Hematoxylin and Eosin (H\&E) Staining}

Direct TTC assay according to our previous work (Li et al., 2012) was used to determine myocardial infarct size. In brief, the heart was transversely cut across both ventricles and sectioned into $2 \mathrm{~mm}$ thick. Sections were incubated at $37^{\circ} \mathrm{C}$ in $1 \%$ TTCphosphate buffer ( $\mathrm{pH} 7.4$ ) for $30 \mathrm{~min}$, following which they were fixed with $4 \%$ paraformaldehyde for $24 \mathrm{~h}$. The non-ischemic myocardium and viable ischemic myocardium were stained red, while the infarcted myocardium appeared pale gray or white. The slices were photographed using a digital camera, and the $\%$ infarction was analyzed using the computerized Image-Pro Plus 6.0 software (Media Cybernetics, Inc., Silver Spring, MD, United States).

The tissues from ecardiac apex were fixed with $4 \%$ buffered paraformaldehyde for paraffin embedding. Sections were cut (by Leica RM2016 Microtome) into $5 \mu \mathrm{m}$ and stained with hematoxylin and eosin ( $\mathrm{H} \& \mathrm{E})$. The sections were examined under light microscope (Nikon Eclipse E100) and micro graphed by Nikon DS-U3 digital camera controller at $200 \times$ magnification.

\section{Drug Analysis}

Plasma concentrations of GA were determined by HPLC-UV method which has been firstly developed and validated. Briefly, HPLC analysis was performed on a Woburn $\mathrm{C}_{18}$ column (250 mm $\times 4.6 \mathrm{~mm}, 5 \mu \mathrm{m}$, Woburn) using LC2010-AT chromatographic system (Shimadzu, Kyoto, Japan). The mobile phase consisted of methano-2\% glacial acetic acid solution (5:95, $\mathrm{v} / \mathrm{v}$ ) at a flow rate of $1 \mathrm{~mL} \mathrm{~min}^{-1}$. The temperature of column was maintained at $30^{\circ} \mathrm{C}$. The detection wavelength was set at $272 \mathrm{~nm}$. 4 -acetamidophenol was used as IS.

To $200 \mu \mathrm{L}$ plasma, $50 \mu \mathrm{L}$ of 4-acetamidophenol (40 $\mu \mathrm{g} \mathrm{mL}^{-1}$ ), $100 \mu \mathrm{L}$ of $1 \mathrm{~mol} / \mathrm{L}$ hydrochloric acid as well as $1 \mathrm{~mL}$ ethyl acetate were added and vortexed for $3 \mathrm{~min}$. Followed by centrifugation at $4000 \mathrm{rpm}$ for $15 \mathrm{~min}, 1 \mathrm{~mL}$ aliquot of the supernatant was transferred and the residue was mixed with $1 \mathrm{~mL}$ of ethyl acetate for another same extraction procedure. Finally, both of the supernatants were combined and evaporated to dryness at $40^{\circ} \mathrm{C}$ under a gentle stream of nitrogen. The dried residue was then reconstituted in $200 \mu \mathrm{L}$ of mobile phase. After being vortexed for $1 \mathrm{~min}$, the content was centrifugated at $12000 \mathrm{rpm}$ for $15 \mathrm{~min}$. The supernatant was then transferred to $2 \mathrm{~mL}$ glass vials and an aliquot of $20 \mu \mathrm{L}$ was injected for analysis.

The calibration curve for GA was linear over the concentration range of $0.025-5 \mu \mathrm{g} \mathrm{mL}^{-1}$ with correlation coefficient (R) greater than 0.9999 . The lower limit of quantification was $0.025 \mu \mathrm{g} \mathrm{mL}^{-1}$ $(\mathrm{S} / \mathrm{N} \geq 10)$. Quality control samples were prepared at $0.078,0.63$, and $2.50 \mu \mathrm{g} \mathrm{mL}^{-1}$. The relative standard deviation (RSD) of intra-day and inter-day precisions was less than $1.09 \%(n=5)$. The relative errors (REs) for assay accuracies were found to be within 0.98 to $2.60 \%$. The mean extraction recoveries at three quality control levels were between 87.31 and $90.02 \%(n=5)$.

\section{Pharmacokinetic Study}

For pharmacokinetic study, the rats were randomly and equally divided into four groups including two normal group and two myocardial infarction (MI) group ( $n=8$ for each group). Normal group was treated by oral administration of GA at doses of 50 and $100 \mathrm{mg} / \mathrm{kg}$, respectively. MI group was inducted with ISO for myocardial injury and were treated by oral administration of GA at 50 and $100 \mathrm{mg} / \mathrm{kg}$, respectively. Dose of GA were selected on the basis of previous studies (Hsu and Yen, 2007; Yousuf and Vellaichamy, 2015; Oyagbemi et al., 2016) and our pilot study. Approximately $300 \mu \mathrm{L}$ (400 $\mu \mathrm{L}$ for the upper limit value) $(\mathrm{NIH}$, 2015) blood samples were collected via retro-orbital sinus and transferred into heparinzed tubes at 0 (prior to dosing, serve as control), 10, 30, 60, 90, 120, 180, 300, 420, 540, and 600 min after oral administration. The technical personnel involved were welltrained in blood draws via retro-orbital sinus such that it imparts minimal pain to the animals. The rats were free access to $0.9 \%$ saline after collection time point (or administered equal volume of $0.9 \%$ saline orally if necessary) for fluid replacement. $200 \mu \mathrm{L}$ of plasma was harvested by centrifuging the blood sample at $4^{\circ} \mathrm{C}$ and $4000 \mathrm{rpm}$ for $15 \mathrm{~min}$, and then stored at $-80^{\circ} \mathrm{C}$ until analysis.

\section{Western Blot Analysis}

Expressions of tight junction (TJ) proteins in small intestine of rats were analyzed as previously described immunoblotting method (Li et al., 2016) using the following antibodies: claudin1, occluding, zo-1, and $\beta$-actin (Cell Signaling Technology, Danvers, MA, United States). Immunoreactive proteins were visualized by enhanced chemiluminescence using ECL kit (Guge Biotechnology, Wuhan, China). The signal intensity was detected and quantified by ChemiScope 5300 Pro System (Clinx Science Instruments, Co., Ltd., Shanghai, China).

\section{Statistical Analysis}

The pharmacokinetic parameters of GA were analyzed through non-compartmental analysis using Drug and Statistics version 2.0 (DAS 2.0, Anhui Provincial Center for Drug Clinical Evaluation) software, including the half-life $\left(\mathrm{T}_{1 / 2}\right)$, mean residence time (MRT), area under the plasma concentration-time curve (AUC), clearance $(\mathrm{CL} / \mathrm{F})$, and volume of distribution $\left(\mathrm{V}_{\mathrm{d}} / \mathrm{F}\right)$. Data were shown as mean \pm standard deviation (mean \pm SD). The difference between groups was analyzed with the Student's $t$-test using GraphPad Prism 6.0 statistical package program (GraphPad Software, Inc., La Jolla, CA, United States). p-Value less than 0.05 was considered as statistically significant.

\section{RESULTS AND DISCUSSION}

Gallic acid is an endogenous polyphenol found abundantly in medicinal plant. In recent years, some pharmacokinetic studies on crude drugs and/or Chinese patented medicines which 
A

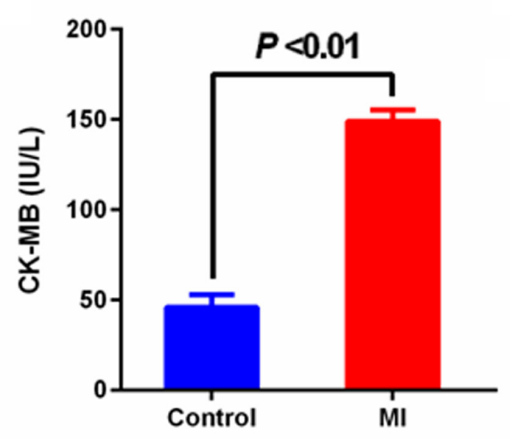

C

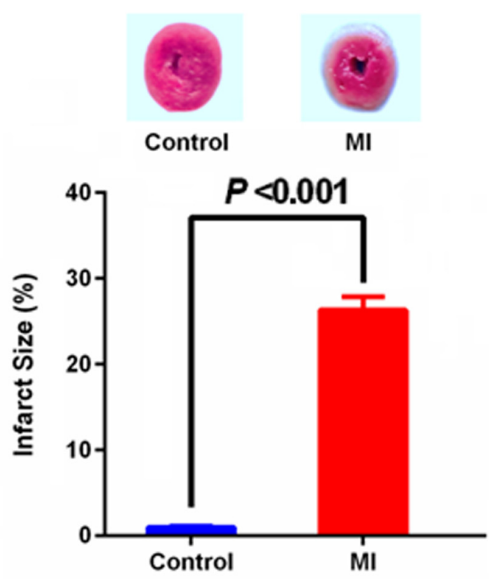

B

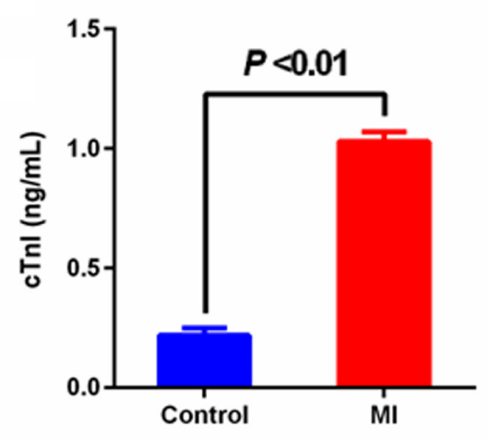

D

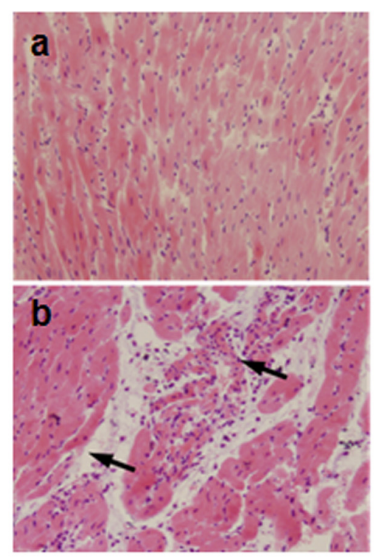

FIGURE 2 | Effect of myocardial infarction (MI) on serum-specific cardiac biomarkers [(A) CK-MB, (B) cTnl; values are expressed as mean \pm SD ( $n=8)]$, (C) myocardial infarct size [normal myocardium was stained red, while pale gray areas indicate infarct areas; values are expressed as mean \pm SD $(n=4)$ ] and histopathologic changes of heart [(D) (a) control, (b) Ml; heart tissues were stained with hematoxylin and eosin and visualized under light microscope at 200× magnification].

content GA has been performed on healthy volunteers (Shahrzad and Bitsch, 1998) and normal animals (Xu et al., 2017; Zhu et al., 2017). However, considerable uncertainty remains due to the lack of comprehensive kinetic profiles after administration of GA monomer in vivo, especially under pathological condition. In the present study, the pharmacokinetic characteristics of GA were investigated in detail for the first time, and they were found to be great difference in normal and ISO-induced myocardial infarcted rats.

Myocardial infarction is a common presentation of IHD. It is the acute condition arising from the critical imbalance between coronary oxygen supply and myocardial demand, resulting in necrosis of the myocardium. ISO in supramaximal doses induces morphological and functional alterations in the heart leading to subendocardial myocardial ischemia, hypoxia and necrosis, which closely resembles local MI-like pathological changes seen in human MI (Li et al., 2016). During MI condition, cardic $\mathrm{CK}-\mathrm{MB}$ and cardiac troponin I (cTnI) were released from the damaged heart tissue into the blood stream. Thus, levels of these specific cardiac biomarkers serve as sensitive indices to assess the severity of MI. As shown in Figure 2, there was a

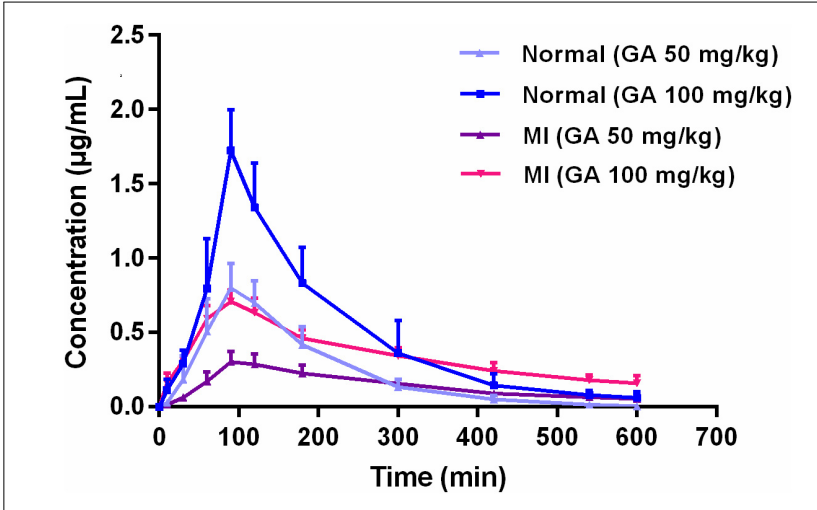

FIGURE 3 | Mean plasma concentration-time profiles of gallic acid in normal and $\mathrm{Ml}$ rats after oral administration of 50 and $100 \mathrm{mg} / \mathrm{kg} \mathrm{GA}$, respectively.

significant $(p<0.001)$ rise observed in the levels of CK-MB and cTnI the serum of group ISO-administered rats in comparison with normal rats, and abnormal histoarchitecture (e.g., loss of 
TABLE 1 | Pharmacokinetic parameters of GA in normal and ISO-induced myocardial infarcted rats after oral administration of 50 and 100 mg/kg GA, respectively (mean $\pm \mathrm{SD}, n=8)$.

\begin{tabular}{|c|c|c|c|c|c|}
\hline \multirow[t]{2}{*}{ Pharmacokinetic parameters } & \multirow[t]{2}{*}{ Units } & \multicolumn{2}{|c|}{ GA (50 mg/kg) } & \multicolumn{2}{|c|}{ GA (100 mg/kg) } \\
\hline & & Normal & MI & Normal & MI \\
\hline $\mathrm{C}_{\max }$ & $\mu \mathrm{g} \cdot \mathrm{mL}^{-1}$ & $0.83 \pm 0.15$ & $0.32 \pm 0.11^{*}$ & $1.71 \pm 0.29$ & $0.72 \pm 0.19^{*}$ \\
\hline $\mathrm{T}_{\max }$ & $\min$ & $90.00 \pm 21.21$ & $102.00 \pm 15.49$ & $85.00 \pm 12.25$ & $96.00 \pm 13.42$ \\
\hline $\mathrm{T}_{1 / 2}$ & $\min$ & $153.65 \pm 19.45$ & $244.51 \pm 26.23^{*}$ & $186.67 \pm 23.97$ & $278.17 \pm 26.86$ \\
\hline $\mathrm{AUC}_{0-\mathrm{t}}$ & $\mathrm{mg} \cdot \min \cdot L^{-1}$ & $137.07 \pm 24.75$ & $83.41 \pm 18.17^{*}$ & $262.55 \pm 22.05$ & $198.99 \pm 24.69$ \\
\hline $\mathrm{AUC}_{0-\infty}$ & $\mathrm{mg} \cdot \min \cdot L^{-1}$ & $150.49 \pm 26.35$ & $121.62 \pm 17.16$ & $280.49 \pm 26.35$ & $300.52 \pm 37.33$ \\
\hline $\mathrm{MRT}_{0-\mathrm{t}}$ & $\min$ & $160.08 \pm 18.58$ & $238.58 \pm 14.89^{*}$ & $176.64 \pm 24.03$ & $274.77 \pm 7.29^{*}$ \\
\hline $\mathrm{MRT}_{0-\infty}$ & $\min$ & $187.97 \pm 21.75$ & $301.21 \pm 42.29 *$ & $211.58 \pm 24.54$ & $331.21 \pm 43.89$ \\
\hline $\mathrm{CL}$ & $L \cdot(\min \cdot k g)^{-1}$ & $0.37 \pm 0.08$ & $0.29 \pm 0.10$ & $0.43 \pm 0.06$ & $0.34 \pm 0.04^{*}$ \\
\hline $\mathrm{V}_{\mathrm{d}}$ & $\mathrm{L} \cdot \mathrm{kg}^{-1}$ & $78.52 \pm 8.51$ & $131.09 \pm 24.16^{*}$ & $89.60 \pm 10.53$ & $113.09 \pm 13.58$ \\
\hline
\end{tabular}

* $p<0.05$ compared with respective normal rats.

myofibrillar alignment and infiltration of inflammatory cells) of heart tissues were also observed in the ISO-induced rats, indicating the leakage and loss of functional integrity and/or necrotic damage of cell membrane. These results were in accordance with the direct staining of myocardial tissue using TTC dye, which forms a red formazan precipitate with the intact dehydrogenase enzyme systems of the viable myocardial tissue, whereas the infracted myocardium lack dehydrogenase activities thus fails to stain with TTC. ISO-induced rats showed increase in myocardial infarct size with less TTC absorbing capacity, indicating that the myocardial injury model was successfully induced thus can largely imitate the pathological condition of MI.

Pharmacokinetics of GA in rat plasma after dosage of oral GA monohydrate of 50 and $100 \mathrm{mg} / \mathrm{kg}$ between normal and ISO-induced myocardial infarcted rats was then compared. The mean plasma concentration-time profiles of GA in normal and MI rats were presented in Figure 3. The main pharmacokinetic parameters of GA were calculated by the non-compartmental model and results were summarized in Table 1. After oral administration, the $\mathrm{T}_{\max }$ for intact $\mathrm{GA}$ in normal rats (90 min for $50 \mathrm{mg} / \mathrm{kg} \mathrm{GA}$ and $85 \mathrm{~min}$ for $100 \mathrm{mg} / \mathrm{kg}$ GA, respectively) and ISO-induced myocardial infarcted rats $(102 \mathrm{~min}$ for $50 \mathrm{mg} / \mathrm{kg}$ GA and $96 \mathrm{~min}$ for $100 \mathrm{mg} / \mathrm{kg}$ GA, respectively) had no significant change, although ISO treated rats showed a slight delay. There were no significant differences in the $T_{1 / 2}$ and $V_{d}$ values when the GA dose was increased from 50 to $100 \mathrm{mg} / \mathrm{kg}$ body weight, while it was worth noting that the $\mathrm{C}_{\max }$ and AUC of GA increased with dose in a dose-proportional manner at the doses of 50 and $100 \mathrm{mg} / \mathrm{kg}$ in both ISO groups and normal group, indicating that the pharmacokinetic behavior of GA could be in a dose-dependent manner. Moreover, it was well to be reminded that $\mathrm{MI}$ could significantly decrease the $\mathrm{C}_{\max }$, $\mathrm{CL}$, and AUC values whereas increase the $\mathrm{T}_{1 / 2}, \mathrm{MRT}$, and $\mathrm{V}_{\mathrm{d}}$ values. $\mathrm{C}_{\max }$ values of $\mathrm{GA}$ were estimated to be $0.32 \mu \mathrm{g} \mathrm{\textrm {mL } ^ { - 1 }}$ (50 mg/kg GA) and $0.72 \mu \mathrm{g} \mathrm{mL} \mathrm{m}^{-1}(100 \mathrm{mg} / \mathrm{kg} \mathrm{GA})$ in $\mathrm{MI}$ rats, which were approximately 2.5 -fold lower than those in the respective dosed normal rats. The $\mathrm{AUC}_{0-\mathrm{t}}$ of $\mathrm{MI}$ rats was also found to be decreased 1.7-fold (50 mg/kg GA) and 1.3-fold (100 mg/kg GA) when compared to respective dosed normal rats. Konishi et al. (2003) have reported that the permeation of GA was not in a polarized manner and was independent of $\mathrm{pH}$, which indicate that the transepithelial transport of GA is permeated via the paracellular diffusion. Interestingly, results of our immunoblotting analysis (Figure 4) showed that MI condition significantly increased the expression of claudin-1, occludin, and zo-1 proteins in the small intestine when compared to normal rats. Claudin-1, occluding and zo-1 belong to the family of proteins that are the most important components of

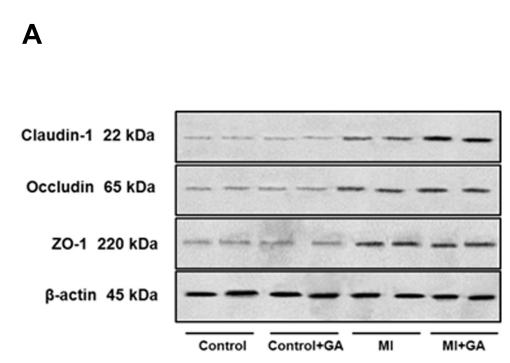

B

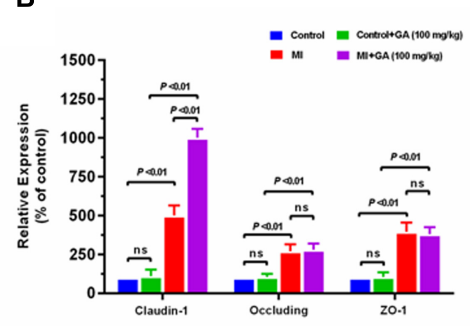

FIGURE 4 | Effect of myocardial infarction and GA (100 mg/kg) on expressions of tight junction proteins in small intestine. Graphs represent (A) immunoblot of small intestine claudin-1, occluding, and zo-1 protein expressions and densitometry analysis of (B) claudin-1, occluding, and zo-1 protein expressions. Tissue samples were harvested after the last time point of blood collection. Values are expressed as mean $\pm \mathrm{SD}(n=4)$; ns, not significant. 
the TJs, where they establish the paracellular barrier that controls the flow of hydrophilic small molecules in the intercellular space between intestinal epithelial cells (Suzuki et al., 2017). Thus, we hypothesized that the poorer permeability through the intestinal epithelial membrane is more likely to be responsible for the decreased values of $\mathrm{T}_{\max }, \mathrm{AUC}_{0-\mathrm{t}}$, and $\mathrm{C}_{\max }$ of GA after MI. Furthermore, significant prolonged $\mathrm{T}_{1 / 2}$ and MRT as well as decreased CL were also registered between normal and MI rats. It has been reported that when MI occurred, the volume of blood plasma was often decreased and drug clearance may also be diminished due to decreased cardiac output and blood flow to the major organs such as liver and kidneys, as well as decreased hepatic drug metabolizing activity (Imanparast et al., 2017). Li et al. (2015) found that MI rats induced by pituitrin had a longer half-life time and MRT of notoginsenoside compared with the normal rats, indicating pathological state of MI might cause variation of absorption and metabolism after drug was orally administered compared with those under the normal state. In addition, the elimination rate of $\mathrm{GA}$ slowed down while $\mathrm{AUC}_{0-\infty}$ showed a slight increase in MI rats, which suggest the drug might cumulate in the pathological state. Therefore, it is necessary to investigate the dynamics of GA to enhance its safety and efficacy during clinical applications.

\section{CONCLUSION}

In summary, our findings suggest that the pharmacokinetic process of GA could be altered in the condition of myocardial ischemia, and the parameters obtained from the normal state are limited and needs to be modified according to the practice. Kinetic features of GA obtained from the present study could be applied as a reference for evaluating its clinical efficacy.

However, it should be mentioned that in the present study approximately $1.5 \%$ of total circulating blood volume (CBV) was taken per time point/rat, which was greater than the volume (1\% CBV) recommended by NIH (NIH, 2015). Reports have addressed that pharmacokinetic behavior of some drugs could be altered by supramaximal fluid loss (Kugelberg et al., 2006),

\section{REFERENCES}

Farías, J. G., Molina, V. M., Carrasco, R. A., Zepeda, A. B., Figueroa, E., Letelier, P., et al. (2017). Antioxidant therapeutic strategies for cardiovascular conditions associated with oxidative stress. Nutrients 9:E966. doi: 10.3390/nu9090966

Hsu, C. L., and Yen, G. C. (2007). Effect of gallic acid on high fat diet-induced dyslipidaemia, hepatosteatosis and oxidative stress in rats. Br. J. Nutr. 98, 727-735. doi: 10.1017/S000711450774686X

Imanparast, A., Fatouraee, N., and Sharif, F. (2017). Comprehensive computational assessment of blood flow characteristics of left ventricle based on in-vivo MRI in presence of artificial myocardial infarction. Math. Biosci. 294, 143-159. doi: 10.1016/j.mbs.2017.10.007

Konishi, Y., Kobayashi, S., and Shimizu, M. (2003). Transepithelial transport of p-coumaric acid and gallic acid in Caco-2 cell monolayers. Biosci. Biotechnol. Biochem. 67, 2317-2324. doi: 10.1271/bbb.67.2317

Kosuru, R. Y., Roy, A., Das, S. K., and Bera, S. (2017). Gallic acid and gallates in human health and disease: do mitochondria hold the key to success? Mol. Nutr. Food Res. doi: 10.1002/mnfr.201700699 [Epub ahead of print]. though some could not (Sue et al., 1989). Whether the lager amount of blood sampling has potential effects (or bias) on the pharmacokinetic behavior of GA is unclear. Studies will be conducted to make this issue clear in the further work. In addition, it should also be note the effects of many drugs could be attributed partially to the action of their metabolites, rather than the direct action of the parent drug (Yasuda et al., 2000). Thus, further investigations are necessary to focus on the absorption mechanism of both GA and its metabolites in gastrointestinal track between normal and pathological conditions.

\section{AUTHOR CONTRIBUTIONS}

ZY and HL conceived and designed the experiments, performed the experiments, and wrote the original manuscript. FS performed the experiments and analyzed the data. Y-CJ and W-MZ analyzed the data, prepared figures, and crafted the final manuscript. YZ and E-JL helped with the experiments and prepared figures. DZ, L-LB, and QY helped with the analysis of data. HL collected data, provided constructive comments, and crafted the manuscript. B-LZ and S-WW conceived and supervised the study. All authors reviewed and approved the final manuscript.

\section{FUNDING}

This work was supported by the National Natural Science Foundation of China (Program No. 81703737), the Basic Research Project of Shaanxi Administration of Traditional Chinese Medicine (Program No. ZYMS012), and the Natural Science Basic Research Plan in Shaanxi Province of China (Program No. 2016JQ8035).

\section{ACKNOWLEDGMENTS}

We thank Ms. Feifei Su and Ms. Xiaoli Gao for their help with the animal experiments of this study.

Kugelberg, F. C., Alkass, K., Kingbäck, M., Carlsson, B., and Druid, H. (2006). Influence of blood loss on the pharmacokinetics of citalopram. Forensic Sci. Int. 161, 163-168. doi: 10.1016/j.forsciint.2006.02.050

Li, H., Song, F., Duan, L. R., Sheng, J. J., Xie, Y. H., Yang, Q., et al. (2016). Paeonol and danshensu combination attenuates apoptosis in myocardial infarcted rats by inhibiting oxidative stress: Roles of Nrf2/HO-1 and PI3K/Akt pathway. Sci. Rep. 6:23693. doi: 10.1038/srep23693

Li, H., Wang, S. W., Zhang, B. L., Xie, Y. H., Yang, Q., Cao, W., et al. (2011). Simultaneous quantitative determination of 9 active components in traditional Chinese medicinal preparation ShuangDan oral liquid by RP-HPLC coupled with photodiode array detection. J. Pharm. Biomed. Anal. 56, 820-824. doi: 10.1016/j.jpba.2011.07.016

Li, H., Xie, Y. H., Yang, Q., Wang, S. W., Zhang, B. L., Wang, J. B., et al. (2012). Cardioprotective effect of paeonol and danshensu combination on isoproterenol-induced myocardial injury in rats. PLoS One 7:e48872. doi: 10.1371/journal.pone.0048872

Li, L. M., Liu, R. X., Guo, J. W., Deng, Z. J., Ren, B., Li, A. R., et al. (2015). In vivo pharmacokinetics of Notoginsenoside R1 in 
ischemia rats after acute myocardial infarction. J. Chin. Med. Mater 9, 1908-1911.

Ma, F., Gong, X., Zhou, X., Zhao, Y., and Li, M. (2015). An UHPLC-MS/MS method for simultaneous quantification of gallic acid and protocatechuic acid in rat plasma after oral administration of Polygonum capitatum extract and its application to pharmacokinetics. J. Ethnopharmacol. 162, 377-383. doi: 10.1016/j.jep.2014.12.044

NIH (2015). Guidelines for Survival Bleeding of Mice and Rats. NIH Animal Research Advisory Committee Guidelines B-2. Available at: https://oacu.oir.nih. gov/sites/default/files/uploads/arac-guidelines/rodent_bleeding.pdf.

Nwokocha, C., Palacios, J., Simirgiotis, M. J., Thomas, J., Nwokocha, M., Young, L., et al. (2017). Aqueous extract from leaf of Artocarpus altilis provides cardio-protection from isoproterenol induced myocardial damage in rats: negative chronotropic and inotropic effects. J. Ethnopharmacol. 203, 163-170. doi: 10.1016/j.jep.2017.03.037

Oyagbemi, A. A., Omobowale, T. O., Saba, A. B., Olowu, E. R., Dada, R. O., and Akinrinde, A. S. (2016). Gallic acid ameliorates cyclophosphamideinduced neurotoxicity in wistar rats through free radical scavenging activity and improvement in antioxidant defense system. J. Diet. Suppl. 13, 402-419. doi: 10.3109/19390211.2015.1103827

Priscilla, D. H., and Prince, P. S. (2009). Cardioprotective effect of gallic acid on cardiac troponin- $\mathrm{T}$, cardiac marker enzymes, lipid peroxidation products and antioxidants in experimentally induced myocardial infarction in Wistar rats. Chem. Biol. Interact. 179, 118-124. doi: 10.1016/j.cbi.2008. 12.012

Shahrzad, S., Aoyagi, K., Winter, A., Koyama, A., and Bitsch, I. (2001). Pharmacokinetics of gallic acid and its relative bioavailability from tea in healthy humans. J. Nutr. 131, 1207-1210. doi: 10.1093/jn/131.4. 1207

Shahrzad, S., and Bitsch, I. (1998). Determination of gallic acid and its metabolites in human plasma and urine by high-performance liquid chromatography. J. Chromatogr. B Biomed. Sci. Appl. 705, 87-95. doi: 10.1016/S0378-4347(97) 00487-8

Stanely, M. P. P., Priscilla, H., and Devika, P. T. (2009). Gallic acid prevents lysosomal damage in isoproterenol induced cardiotoxicity in Wistar rats. Eur. J. Pharmacol. 615, 139-143. doi: 10.1016/j.ejphar.2009.05.003
Sue, D., Salazar, T. A., Turley, K., and Guglielmo, B. J. (1989). Effect of surgical blood loss and volume replacement on antibiotic pharmacokinetics. Ann. Thorac. Surg. 47, 857-859. doi: 10.1016/0003-4975(89)90020-9

Suzuki, R., Katsuno, T., Kishimoto, Y., Nakamura, R., Mizuta, M., Suehiro, A., et al. (2017). Process of tight junction recovery in the injured vocal fold epithelium: Morphological and paracellular permeability analysis. Laryngoscope doi: 10.1002/lary.26959 [Epub ahead of print].

Xu, C., Yu, Y., Ling, L., Wang, Y., Zhang, J., Li, Y., et al. (2017). A C8-Modified Graphene@mSiO2 composites based method for quantification of Gallic Acid in rat plasma after oral administration of Changtai Granule and its application to pharmacokinetics. Biol. Pharm. Bull. 40, 1021-1028. doi: 10.1248/bpb.b1700015

Yasuda, T., Inaba, A., Ohmori, M., Endo, T., Kubo, S., and Ohsawa, K. (2000). Urinary metabolites of gallic acid in rats and their radical-scavenging effects on 1,1-diphenyl-2-picrylhydrazyl radical. J. Nat. Prod. 63, 1444-1446. doi: $10.1021 / \mathrm{np} 0000421$

Yousuf, M. J., and Vellaichamy, E. (2015). Protective activity of gallic acid against glyoxal -induced renal fibrosis in experimental rats. Toxicol. Rep. 2, 1246-1254. doi: 10.1016/j.toxrep.2015.07.007

Zhu, H., Liu, X., Zhu, T. T., Wang, X. L., Qin, K. M., Pei, K., et al. (2017). UHPLCMS/MS method for the simultaneous quantitation of five anthraquinones and gallic acid in rat plasma after oral administration of prepared rhubarb decoction and its application to a pharmacokinetic study in normal and acute blood stasis rats. J. Sep. Sci. 40, 2382-2389. doi: 10.1002/jssc.201700166

Conflict of Interest Statement: The authors declare that the research was conducted in the absence of any commercial or financial relationships that could be construed as a potential conflict of interest.

Copyright (®) 2018 Yu, Song, Jin, Zhang, Zhang, Liu, Zhou, Bi, Yang, Li, Zhang and Wang. This is an open-access article distributed under the terms of the Creative Commons Attribution License (CC BY). The use, distribution or reproduction in other forums is permitted, provided the original author(s) and the copyright owner are credited and that the original publication in this journal is cited, in accordance with accepted academic practice. No use, distribution or reproduction is permitted which does not comply with these terms. 